\title{
Heat and Mass Transfer in Partially-Layered Cavity with Inner Square conductive Body
}

\author{
Hassan S. Ghalib, Muneer A. Ismael * \\ Mechanical Engineering Department, Engineering College, University of Basrah, Basrah, Iraq
}

\begin{abstract}
This paper investigates the double diffusive natural convection in a partially porous layered enclosed cavity with a thermally conductive square body. The horizontal walls are thermally insulated, the left wall adds heat isothermally into the porous layer, while the right wall is cooled isothermally. The center of the square conductive body is positioned in the center of the cavity in such a way it lays on the porous-fluid interface. The governing equations have been solved using up-wind scheme finite difference method. The Parndtl number, thermal conductivity ratio of the body to fluid, Darcy number, aspect ratio of the square body to the cavity sides have fixed at $6.26,1,10^{-3}, 0.5$, respectively. The study has been governed by three parameters namely, Lewis number $(\mathrm{Le}=1-50)$, buoyancy ratio $(-10-10)$, and Rayleigh number $\left(10^{3}-\right.$ $\left.10^{6}\right)$. The results have showed that the mass diffusivity ratio, which takes into account non-unity tortuosity ratio $\left(\mathrm{D}_{\text {eff }} / \mathrm{D}=\right.$ 0.53 ) has a significant effect on the mass transfer than the unity value. It is found also that Sherwood number is minimal when the buoyancy ratio equals to -0.5 , otherwise, it increases with increasing the absolute value of the buoyancy ratio.
\end{abstract}

Keywords: Double-diffusive; partitioned cavity; porous medium; inner body; finite difference.

\section{Introduction}

Accompaniment of many technical applications, heat transfer processes occur simultaneously with mass transfer processes. Mass transfer specifically refers to the relative motion of species in a mixture due to concentration gradients. This subject subsumes to applications in geothermal reservoirs, petroleum extraction, home humidifiers, chemical catalytic reactors, prevention of subsoil water pollution, nuclear reactors, underground diffusion of nuclear wastes and other contaminants, and porous material regenerative heat exchangers [1]. The heat and mass transfer (double diffusive) in enclosed cavities composed of porous layer confined or superposed by a clear fluid are studied extensively due to their vital relevant in engineering and industry. Baytas et al. [2] investigated the double diffusive natural convection between a saturated porous layer and an overlying fluid layer in an enclosure using the nonDarcy flow model. Hirata et al. [3] studied the linear stability analysis of thermosolutal natural convection in superposed fluid and porous layers. Islam et al. [4] investigated a double diffusive

${ }^{*}$ Corresponding author. Tel.: +9647801043835

Fax: +9647801043835; E-mail: muneerismael@yahoo.com

(C) 2016 International Association for Sharing Knowledge and Sustainability

DOI: $10.5383 /$ ijtee. 13.01 .010 natural convection in a two dimensional brine saturated porous media, subjected to vertical concentration and temperature gradients. Hadidi et al. [5] analyzed numerically the heat and mass transfer generated in horizontal partially porous enclosure. Their results showed that the presence of the porous layer has strong effects on the heat and mass transfer and the modification of the flow structure. Mharzi et al. [6] studied the natural thermosolutal convection in an elongated enclosure of horizontal axis, partitioned by a vertical porous layer. Their results showed that the heat transfer is more influenced by the thermal conductivity ratio, while the solutal transport is essentially sensitive to the solutal diffusivity ratio. However, when thermal and solutal buoyancy forces $N$ were opposed to each other $(N<0)$, the increase of Lewis number produces an important reduction of the heat exchange average Nusselt number. Bennacer et al. [7] analyzed numerically the double diffusive natural convection in an enclosure fitted with two symmetrical porous layers confining a fluid layer. Gobin et al. [8] investigated a natural convection driven by combined thermal and solutal buoyancy forces in a binary fluid. They confirmed that the presence of the porous layer has a strong influence on the modification of the flow structure and the consequent modifications of the heat transfer. Outaleb et al. [9] 
studied numerically the double diffusive convection in a partially porous cavity with partially permeable walls under the combined buoyancy effects of thermal and mass diffusion. Hadidi et al. [10] studied a double diffusive natural convection heat and mass transfer in a two-dimensional porous media tilted cavity. Rahli and Bouhadef [11] analyzed numerically the double diffusive convection in inclined partially porous cavity, with partially permeable walls. Hadidi and Bennacer [12] investigated the problem with two and three-dimensional flows.

Studies focused on the insertion of conductive or adiabatic bodies inside enclosure can be found in the followings. House et al. [13] performed a study of natural convection in a differentially heated square enclosure containing a square conducting body. Ha and Jung [14] conducted a numerical study in three-dimensional heat transfer and flow characteristics of natural convection in a vertical cubic enclosure when differentially heated. Lee and $\mathrm{Ha}$ [15] investigated natural convection in horizontal layer of fluid with a conducting body in the interior, heated from below and cooled from above walls. Braga and Lemos [16] presented numerical solutions for steady laminar and turbulent natural convection within a square cavity filled by a fixed amount of conducting solid material using a continuum model. Both the continuum and the porous continuum models gave higher values for the Nusselt number when turbulence was considered, being macroscopic solutions more sensitive to the inclusion of a turbulence model. In other study, Braga and Lemos [17] investigated numerically heat transfer across a square cavity partially filled with a fixed amount of a conducting solid shaped with two different types of geometry. Merrikh and Lage [18] investigated numerically natural convection within a differentially heated heterogeneous square enclosure consisting of several disconnected and conducting solid blocks within a saturated fluid. For a number of solid blocks bigger than the minimum number necessary for flow switch, the heat transfer across the enclosure was enhanced as the fluid to-solid thermal conductivity ratio increases. Das and Reddy [19] investigated numerically the natural convection flow in a tilted square enclosure with a centered internal conducting square block. Zhao et al. [20] studied a natural convection heat transfer and fluid flow in a vertical enclosure subject to periodic temperature boundary condition imposed at the right sidewall with a conducting body placed at the center of the enclosure. Zhao et al. [21] in other study investigated the conjugate heat transfer in enclosures encircled with finite walls or with centered solid body. Varol [22] investigated numerically, natural convection in porous media filled cavity. The conducting body inserted in triangular cavity heated from horizontal wall, the vertical wall is adiabatic and the inclined wall is kept cold. Their results showed that the heat transfer and fluid flow were affected by the location of the conducting body. They showed that heat transfer increased with increasing thermal conductivity $k$ and Rayleigh number due to available energy into the system. For a higher value of thermal conductivity, heat transfer becomes constant.

In accordance to the above literature survey, it can be concluded that the double diffusive natural convection in a vertically partitioned-porous cavity with inner fixed conductive body has not been investigated yet. Hence, the scope of this paper is the numerical analysis of natural convection heat and mass transfer in partially layered square cavity with inner thermally conductive square body.

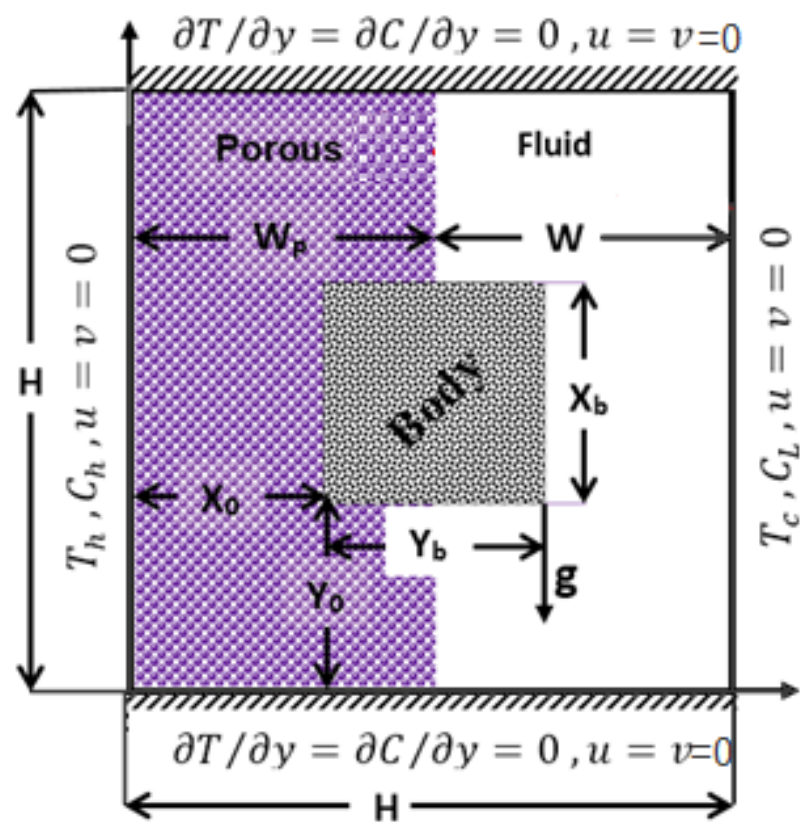

Fig. 1. Physical domain and coordinates system

\section{Mathematical Modelling}

The considered problem is shown schematically in Fig. 1. It is a square cavity with side length $H$. A vertical porous layer of thickness $W_{p}$ is localized on the left part of the cavity and saturated with a fluid. This fluid fills the remainder of the cavity $\left(H-\mathrm{W}_{\mathrm{p}}\right)$. A thermally conductive square body is centered inside the cavity. The left wall of the cavity which is in contact with the porous layer is heated isothermally, kept at $\mathrm{T}_{\mathrm{h}}$ and $\mathrm{C}_{\mathrm{h}}$ (high precise concentrations), while the right wall of the cavity is cooled isothermally, kept at $T_{C}$ and $C_{L}$ (low precise concentrations). The assumptions used to simplify the proposed model are; the flow is two-dimensional, incompressible, laminar, and steady state. All outer boundaries are fixed and impermeable, while the vertical interface between nanofluid and porous layers is permeable. Regarding the porous layer, uniform and undeformable pores between the solid matrixes are assumed. A thermal equilibrium is assumed between the fluid filling pores and the solid matrix. Constant physical properties are assumed except the density where it varies with temperature according to Boussinesq approximation. The energy dissipation and radiation of surface are negligible. Darcy-Brinkman model is invoked to represents the convection within the porous layer, where in this model there is no problem in the order of the matched momentum equation in the fluid/ porous.

Based on the stream function-vorticity formulation [23], the dimensionless governing equations are:

For porous layar

Continuity:

$$
\frac{\partial^{2} \Psi_{p}}{\partial X^{2}}+\frac{\partial^{2} \Psi_{p}}{\partial Y^{2}}=-\Omega_{p}
$$

Momentum:

$$
\begin{gathered}
\frac{\partial \Psi_{P}}{\partial Y} \frac{\partial \Omega_{p}}{\partial X}-\frac{\partial \Psi_{P}}{\partial X} \frac{\partial \Omega_{P}}{\partial Y}=\varepsilon \operatorname{Pr}\left[\frac{\partial^{2} \Omega_{P}}{\partial X^{2}}+\frac{\partial^{2} \Omega_{P}}{\partial Y^{2}}\right]-\frac{P r}{D a} \Omega_{p}+ \\
\varepsilon^{2} \operatorname{RaPr} \frac{\partial \theta}{\partial X}+\varepsilon^{2} \operatorname{RaPrN} \frac{\partial C}{\partial X}
\end{gathered}
$$


Energy:

$$
\frac{\partial \Psi_{p}}{\partial Y} \frac{\partial \theta_{p}}{\partial X}-\frac{\partial \Psi_{p}}{\partial X} \frac{\partial \theta_{P}}{\partial Y}=\frac{\alpha_{e f f}}{\alpha_{f}}\left[\frac{\partial^{2} \theta_{P}}{\partial X^{2}}+\frac{\partial^{2} \theta_{p}}{\partial Y^{2}}\right]
$$

Mass

$\frac{\partial \Psi_{p}}{\partial Y} \frac{\partial C_{p}}{\partial X}-\frac{\partial \Psi_{p}}{\partial X} \frac{\partial C_{P}}{\partial Y}=\frac{D_{e f f}}{D_{f}} \frac{1}{L_{e}}\left[\frac{\partial^{2} C_{P}}{\partial X^{2}}+\frac{\partial^{2} C_{p}}{\partial Y^{2}}\right]$

For fluid:

Continuity:

$$
\frac{\partial^{2} \Psi_{f}}{\partial X^{2}}+\frac{\partial^{2} \Psi_{f}}{\partial Y^{2}}=-\Omega_{f}
$$

Momentum:

$$
\begin{aligned}
& \frac{\partial \Psi_{f}}{\partial Y} \frac{\partial \Omega_{f}}{\partial X}-\frac{\partial \Psi_{f}}{\partial X} \frac{\partial \Omega_{f}}{\partial Y}=\operatorname{Pr}\left[\frac{\partial^{2} \Omega_{f}}{\partial X^{2}}+\frac{\partial^{2} \Omega_{f}}{\partial Y^{2}}\right]+\operatorname{RaPr} \frac{\partial \theta_{f}}{\partial X}+ \\
& \operatorname{RaPrN} \frac{\partial C_{f}}{\partial X}
\end{aligned}
$$

Energy:

$$
\frac{\partial \Psi_{f}}{\partial Y} \frac{\partial \theta_{f}}{\partial X}-\frac{\partial \Psi_{f}}{\partial X} \frac{\partial \theta_{f}}{\partial Y}=\left[\frac{\partial^{2} \theta_{f}}{\partial X^{2}}+\frac{\partial^{2} \theta_{f}}{\partial Y^{2}}\right]
$$

Mass:

$$
\frac{\partial \Psi_{f}}{\partial Y} \frac{\partial C_{f}}{\partial X}-\frac{\partial \Psi_{f}}{\partial X} \frac{\partial C_{f}}{\partial Y}=\frac{1}{L_{e}}\left[\frac{\partial^{2} C_{f}}{\partial X^{2}}+\frac{\partial^{2} C_{f}}{\partial Y^{2}}\right]
$$

\section{For conductive body:}

$$
\frac{\partial^{2} \theta_{b}}{\partial X^{2}}+\frac{\partial^{2} \theta_{b}}{\partial Y^{2}}=0
$$

Where Prandtl number; $\operatorname{Pr}=\frac{v_{f}}{\alpha_{f}}$, Thermal Rayleigh number; Ra $=\frac{g \beta_{\mathrm{T}, \mathrm{f}} \Delta \mathrm{TH}^{3}}{\alpha_{\mathrm{f}} \mathrm{v}_{\mathrm{f}}}$, Darcy number; $\mathrm{Da}=\frac{\mathrm{K}}{\mathrm{H}^{2}}$, Lewis number; Le $=\frac{\alpha_{\mathrm{f}}}{D_{\mathrm{f}}}$, Buoyancy ratio; $N=\frac{\beta_{\mathrm{C}} \Delta \mathrm{C}}{\beta_{\mathrm{T}} \Delta \mathrm{T}}$

The conditions on the outer dimensionless boundaries of the cavity are;

1- On the left wall:

$\Psi=0, \theta=1, C=1, \Omega=-\left(\frac{\partial^{2} \Psi}{\partial X^{2}}\right)$ at $X=0$

2- On the right wall:

$\Psi=0, \theta=0, C=0, \Omega=-\left(\frac{\partial^{2} \Psi}{\partial X^{2}}\right) \quad$ at $X=1$

3- On the bottom and top wall:

$\Psi=0, \quad \partial \theta / \partial Y=0, \partial C / \partial Y=0, \Omega=-\left(\frac{\partial^{2} \Psi}{\partial Y^{2}}\right)$ at $Y=0,1$

The interface boundary solution are derived from equating (continuity) of tangential and normal velocities, shear stress, temperature, mass and the heat flux across the interface, and assuming the same dynamic viscosity $\left(\mu_{\mathrm{eff}}=\mu \mathrm{f}\right)$ [24], in both layers. Hence, the porous-fluid interface conditions can be written as;

$$
\begin{array}{ll}
\theta_{P}=\theta_{f}, & \frac{\partial \theta_{f}}{\partial X}=\frac{k_{e f f}}{k_{f}} \frac{\partial \theta_{p}}{\partial X} \\
C_{P}=C_{f}, & \frac{\partial c_{f}}{\partial X}=\frac{D_{e f f}}{D_{f}} \frac{\partial C_{p}}{\partial X} \\
\Psi_{P}=\Psi_{f}, & \frac{\partial \Psi_{f}}{\partial X}=\frac{\partial \Psi_{P}}{\partial X} \\
\Omega_{P}=\Omega_{f}, & \frac{\partial \Omega_{f}}{\partial X}=\frac{\partial \Omega_{P}}{\partial X}
\end{array}
$$

Body interface, fluid side

$$
\begin{aligned}
& \theta_{b}=\theta_{f}, \quad \frac{\partial \theta_{f}}{\partial n}=\frac{k_{b}}{k_{f}} \frac{\partial \theta_{b}}{\partial n} \\
& \frac{\partial C_{f}}{\partial n}=0 \\
& \Omega_{P}=-\frac{\partial^{2} \Psi}{\partial n^{2}}
\end{aligned}
$$

Body interface, for porous side

$$
\begin{aligned}
& \theta_{b}=\theta_{p}, \quad \frac{\partial \theta_{p}}{\partial n}=\frac{k_{b}}{k_{e f f}} \frac{\partial \theta_{b}}{\partial n} \\
& \frac{\partial C_{p}}{\partial n}=0 \\
& \Psi_{b}=0, \Omega_{P}=\frac{\partial^{2} \Psi}{\partial n^{2}}
\end{aligned}
$$

The local Nusselt number is defined as follows:

$$
N u=\frac{h H}{k}=\frac{-\frac{\partial T}{\partial x} H}{\left(T_{h}-T_{I}\right)}=-\frac{k_{e f f}}{k_{f}} \frac{\partial \theta}{\partial X}
$$

The average Nusselt number along the left wall is:

$$
N u_{a v}=\int_{0}^{1} N u d y
$$

The local Sherwood number is defined as follows:

$$
S h=-\frac{\partial C}{\partial X}
$$

Average Sherwood number

$$
S h_{a v}=\int_{0}^{1} \operatorname{Sh} d y
$$

\section{Numerical implementation and validations}

\subsection{Numerical method}

The square domain has been discredited into $\mathrm{Nx} \times \mathrm{Ny}$ grids. The governing equations (1) to (9) were solved numerically using central finite difference method, Gauss- Seidel iteration procedure with Successive Under Relaxation (SUR) method is followed in the solution. The convective terms of the momentum and energy equations were treated by the up-wind scheme to attain the solution stability. The stream function, vorticity, mass and dimensionless temperature are calculated from continuity, momentum, mass and energy equations, respectively. A FORTRAN in-house computer programmed has been built to achieve the numerical solution.

\subsection{Validations}

\subsubsection{Grid Independency Test}

An extensive mesh testing procedure has been conducted to guarantee a grid independent solution. In Fig. 2, various mesh sizes were explored for the case of $\mathrm{Da}=10^{-3}, \mathrm{Ra}=10, \mathrm{Le}=50$, $\mathrm{N}=1, \mathrm{Pr}=6.26, \mathrm{~W}_{\mathrm{p}}=0.5, \mathrm{~A}=0.3$ and $\mathrm{Kr}=1$. The numerical solution was tested for grid independence by calculating the average Nusselt number and Sherwood number on the heated vertical wall. Based on the relevant criteria (solution accuracy and the time consumed by the processor), a grid size of $91 \times 91$ ensures these criteria. 

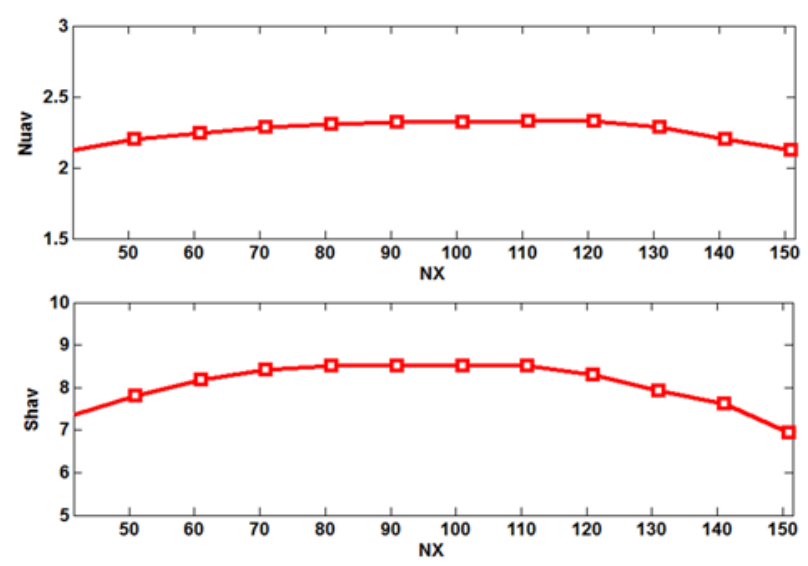

Fig. 2. Testing Grid, $\mathrm{Ra}=10, \mathrm{Le}=50, \mathrm{~N}=1, \mathrm{Pr}=6.26, \mathrm{~W}_{\mathrm{p}}=0.5$, Da $=10, \mathrm{Kr}=5$

\subsubsection{Comparisons with Others}

The present numerical solution was further validated by comparing the present numerical solution results for $\mathrm{Ra}=10^{6}, \mathrm{~N}$ $=10, \operatorname{Pr}=10, W p=0.1$ and aspect ratio (ratio between height to width of cavity $(\mathrm{H} / \mathrm{L})=2$ and different other parameters with the numerical results of Gobin et al. [8]. The tests are presented as the variation of average Nusselt number with different Darcy and Lewis numbers. As shown in Figs. 3, the present numerical solution is in good agreement with Gobin et al. [8]. In accordance to these two validations, the present code can be used to obtain the results with high confidence level.

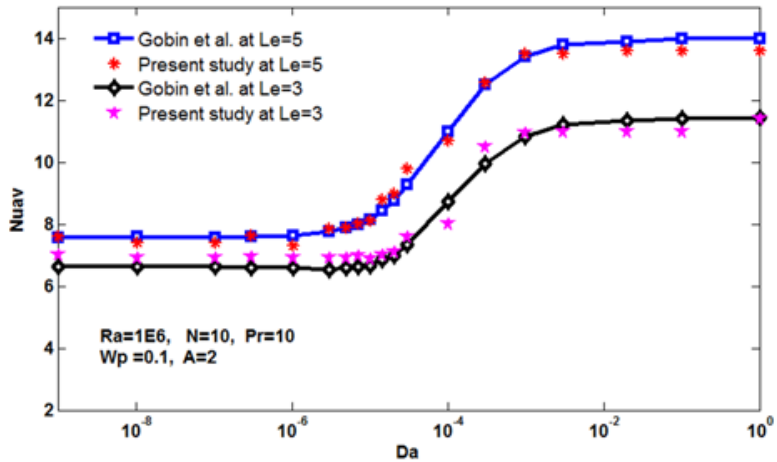

Fig. 3. Comparison of average Nusselt number with Gobin et al. [8].

\section{Results and discussion}

The results of the present paper are to be illustrated graphically by contour maps of stream function, isotherms and isoconcentration, and Nusselt and Sherwood numbers averaged along the left hot vertical wall. The parameters affecting the convection inside the present composite cavity are; Raleigh number Ra, Darcy number Da, Lewis number Le, buoyancy ratio $\mathrm{N}$, conductivity ratio $\mathrm{Kr}$, and the position of the inner conductive body. For brevity, the ranges of selective parameters are as follows: $\mathrm{Ra}=10^{3}-10^{6}, \mathrm{Le}=1-$ $50, \mathrm{~N}=-10-10$. The other parameters are fixed at: $\mathrm{Da}=10^{-3}$, $\operatorname{Pr}=6.26, \mathrm{Kr}=1$, and the conductive body is positioned at the cavity center.
Physically, Rayleigh number represents the ratio of the thermal buoyancy to viscous forces. Lewis number is the ratio of thermal diffusivity to mass diffusivity, and the buoyancy ratio reflects the solutal to thermal buoyancy.

In this study, the fluid is considered as pure water $(\mathrm{Pr}=$ 6.26). The porosity value of the porous layer $\varepsilon$ is fixed at $\varepsilon=$ 0.398 which corresponds to $3 \mathrm{~mm}$ diameter glass beads having a thermal conductivity of $0.845 \mathrm{~W} / \mathrm{m} . \mathrm{k}$ [23]

\subsection{Influence of the buoyancy ratio $\mathrm{N}$}

Before starting with discussion of contour maps, it is worth mentioning that the sign of the streamlines determine the direction of streamlines, i.e. the negative streamlines means clockwise $(\mathrm{CW})$ rotation and positive streamlines means counter-clockwise $(\mathrm{CCW})$ rotation. It is also worth noting that the double diffusive convection in the present study results from the interaction of two mechanisms; heat transfer due to the temperatures difference and the mass transfer due to the concentrations difference.

Figure 4 presents the effect of the bouncy ratio $N$ on the flow fields (left column), the thermal field (mid column) and the concentration field (right column) for $\mathrm{Ra}=10^{5}$ and $\mathrm{Le}=$ 10. The existing of the inner solid square body generates an annulus flow path. This path comprises four grooves, these; the left vertical groove (within the porous medium), the right vertical groove (within the fluid layer), the upper and lower grooves (partly in porous and partly in fluid layers). Generally, when the fluid is heated from left wall, flow moves to right and buoyancy force rises it up within the left groove towards the adiabatic upper wall of the cavity, and then flow moves toward the right wall. As such a $\mathrm{CW}$ rotating vortex is formed. This flow behavior is very clear when $N=3$ (Fig. 4a), where the action of the solutal buoyancy is combined in aiding manner with natural convection effect (positive $N$ ). The flow circulates in a primary vortex within the whole annulus with multi cores. The denes streamlines close to the solid boundaries imply to the intensified flow there. The isotherms (middle column) are purely vertical in the square body (purely heat conduction), approximately vertical in the vertical grooves and tend to be horizontal within the horizontal grooves. This means that the heat transfers horizontally in the vertical grooves and almost vertically within the horizontal grooves. The isoconcentrations lines (right column) are denser close to the left wall. In the horizontal upper groove, the isoconcentrations imply to that, the species falls down from the upper wall of the cavity. However, the combined action resulting from the high buoyancy ratio $(N=3)$ and relatively high Rayleigh number $\left(R a=10^{5}\right)$ leads to high mass transfer as can be seen from the thin solutal boundary layer close to the left wall. For purely natural convection $(N=0$, Fig. $4 \mathrm{~b})$, the flow circulates under the effect of natural convection only. Therefore, the thermal buoyancy is insufficient to strongly circulate the flow within the left groove because the high resistance exerted by the porous layer. Thus, the flow intensity looks very weak within the porous layer. The isoconcentrations appear in disturbed form, while the 
isotherms remain unchanged. When $N=-3.0$ (Fig. 4c) the opposing solutal buoyancy is completely dominant. This can be charachterized by the single $\mathrm{CCW}$ vortex that occupies all the flow passages. The isotherm is almost vertical since heat is transferred by conduction in porous layer. In fluid layer, there is a slight change in the isotherms, which show a bit convective heat transfer due to the skewed isotherms in the horizontal grooves. While the mass transfer increases according to the initiation of the solutal boundary layer, close the upper part of the left wall. Fig. 5 illustrates the influence of the buoyancy ratio $N$ on the average Sherwood number in aiding and opposing modes. It is clear that for $\mathrm{N} \geq 0$ that the average Sherwood number is an increasing function with respect of $N$. The increasing average Sherwood number in aiding flow is representative to the coincided effects of the solutal buoyancy force and thermal buoyancy force in natural convection that causes increase of the mass transfer in the cavity. The minimum value of Sherwood number is recorded at $N=-0.5$, this means that solutal and thermal buoyancies have equivalent but opposite effects. For $\mathrm{N}>-0.5$ the solutal buoyancy predominates over the thermal buoyancy, so the Sherwood number increases again with $N$. Although the apparent symmetry of Sh number about $N=$ -0.5 , but for a given absolute buoyancy ratio, the aiding one gives greater mass transfer than the opposing one.
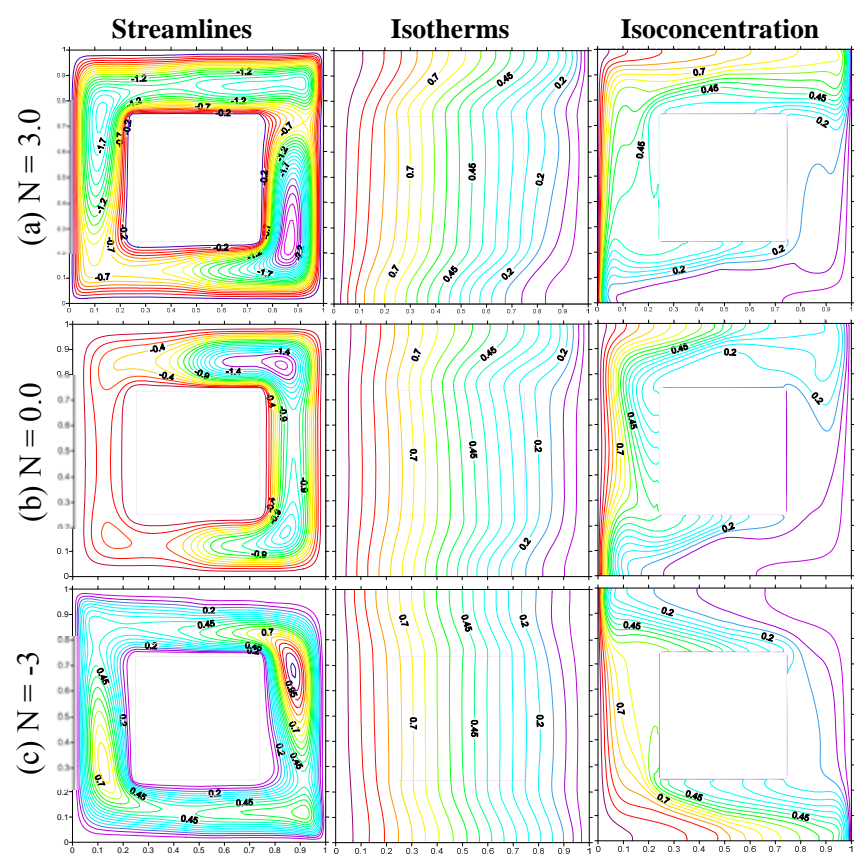

Fig. 4. Effects of buoyancy ratio $\mathrm{N}$ on the streamlines, isotherms and isoconcentrations contours for $\mathrm{Ra}=10^{5}, \mathrm{Le}=10$, and different $\mathbf{N}$

\subsection{Influence of Lewis number}

The effect of Lewis number is inspected by fixing the others parameters constants at $\mathrm{Ra}=10^{4}, N=1$, and depicted in Fig. 6. As mentioned above, Lewis number Le governs the ratio of thermal diffusivity to mass diffusivity.

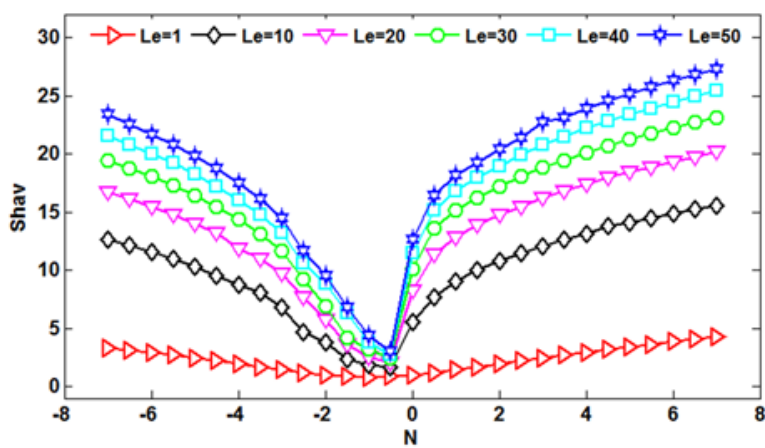

Fig. 5. Effect of buoyancy ratio on Sherwood number at $\mathbf{R a}=10^{5}$

Therefore, increasing Le can be interpreted either by the dominance of the thermal diffusivity which restricts the convective heat transfer or by diminishing the mass diffusivity with respect to thermal diffusivity. As a results, it is clear from Fig. 6 that convective currents weakens where the streamlines at $\mathrm{Le}=1$ are dense and strong throughout the cavity keeping in mind it stronger within the fluid layer (right groove) due to the less flow resistance. However, when Le increased to 50 (Fig. 6c), the flow intensity within the porous layer is notably reduced which is characterized by less density streamlines. It is found that at $\mathrm{Le}=1$, the value of maximum absolute stream function is $|\psi|_{\max }=0.383$, while at $\mathrm{Le}=50$, it decreases to $|\psi|_{\max }=0.24$. The isotherms looks unaffected by increasing Le number, this may because that the buoyancy force is already low $(\mathrm{Ra}=$ $10^{4}$ ). At $\mathrm{Le}=1.0$, the equivalent thermal and mass diffusivities results in a vertical isoconcentrations lines especially in the upper and lower grooves. When Le increases to 30 and further to 50 , thinner solutal boundary layers form close to the vertical cavity walls, while the dominant thermal diffusivity brings the isoconcentrations line to be mostly horizontal within the upper groove.

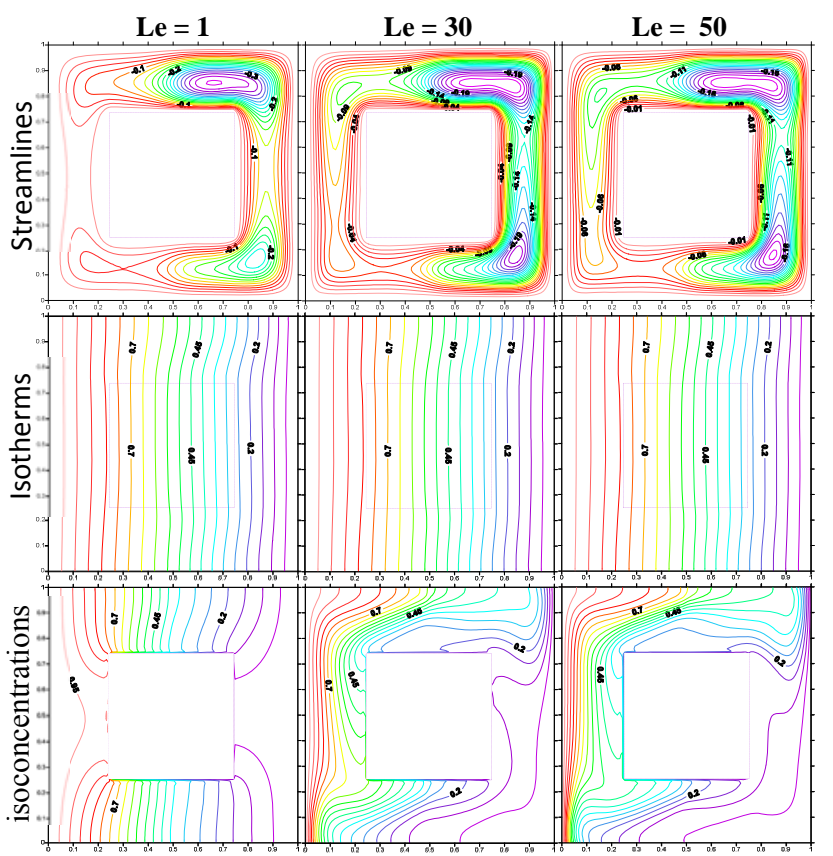

Fig. 6. Streamlines, isotherms, and isoconcentrations contours for selected values of Lewis number, at $\mathrm{Ra}=10^{4}$ and $\mathrm{N}=1.0$. 


\subsection{Influence of Rayleigh number}

It is well known that the effect of Rayleigh number is to increase the buoyancy force over the viscous force; however, it is studied here to inspect its effect on the mass transfer as well. Figure 7 presents this effect for $\mathrm{Le}=10$ and $N=1$. The streamlines variations patterns can be characterized by their intensity strengthening with $\mathrm{Ra}$. It is recorded that the maximum absolute stream function $|\psi|_{\max }$ strengthens according as $0.24,2.539$, and 12.103 when $\mathrm{Ra}$ increased from $10^{4}, 10^{5}$, to $10^{6}$, respectively. The isotherms are vertically distributed at $\mathrm{Ra}=10^{4}$ as an indication to the conduction dominance. At $\mathrm{Ra}=10^{5}$, a bit convective heat transfer takes place at the horizontal grooves. As $\mathrm{Ra}$ increases further to $10^{6}$, the existing of the relatively thin thermal boundary layer and the stratification behavior of the isotherms, especially in the horizontal grooves, imply to the dominance of the convective heat transfer.

If the mechanical movement of the convective currents is strong enough, they can carry the species. This is clear in Fig. 7 (lower row), where the isoconcentrations lines become denser (thinner solutal boundary layer) close the solid walls. Moreover, the concentration gradients settle close to the solid boundary while the middle of the cavity is free of mass transfer.

Figures 8 and 9 present the variations of the Nusselt and Sherwood numbers, respectively, for two values of solutal diffusivity ratios, $\mathrm{DR}=1$ (as assumed in most previous studies) and $\mathrm{DR}=0.53$ (as taken in this paper). The later value takes into account the tortuous path of porous medium. However, these two values have been inspected with various Lewis and Rayleigh numbers. Intuitively, the tortuous path restricts the fluid motion within the porous media, therefore, the average Nusselt number decreases when setting DR $=0.53$ as shown in Fig. 8. This decrement arises at higher Rayleigh number and lower Lewis number, where in these two situations the natural convection is very high. However, for low Rayleigh number and high Lewis number, the effect of DR vanishes. On the other hand, the average Sherwood number rises considerably with decreasing the diffusivity ratio to $\mathrm{DR}=0.53$ as shown in Fig. 9. The reason of this refers to that when the mass diffusivity decreases, the concentration gradients increase within limited situations, which in turn increases the Sherwood number. Hence, the rate of mass transfer is higher than that of heat transfer in the cavity and introducing tortuous path effect within the porous layer will boost extra restriction to the fluid motion. Hence, the real value of diffusivity ratio $\mathrm{DR}=0.53$ was adopted in all previous results.

Moreover, Figs. 8 and 9 demonstrate that the Nusselt number undergoes the effect of Le up to $\mathrm{Le}=10$, otherwise, the thermal buoyancy effect becomes immune against Le number. Meanwhile, the average Sherwood number increases continuously with Le number.

The effect of increasing Ra number can strongly enhance the convective heat transfer, and as a result, the mass transfer can be enhanced too.

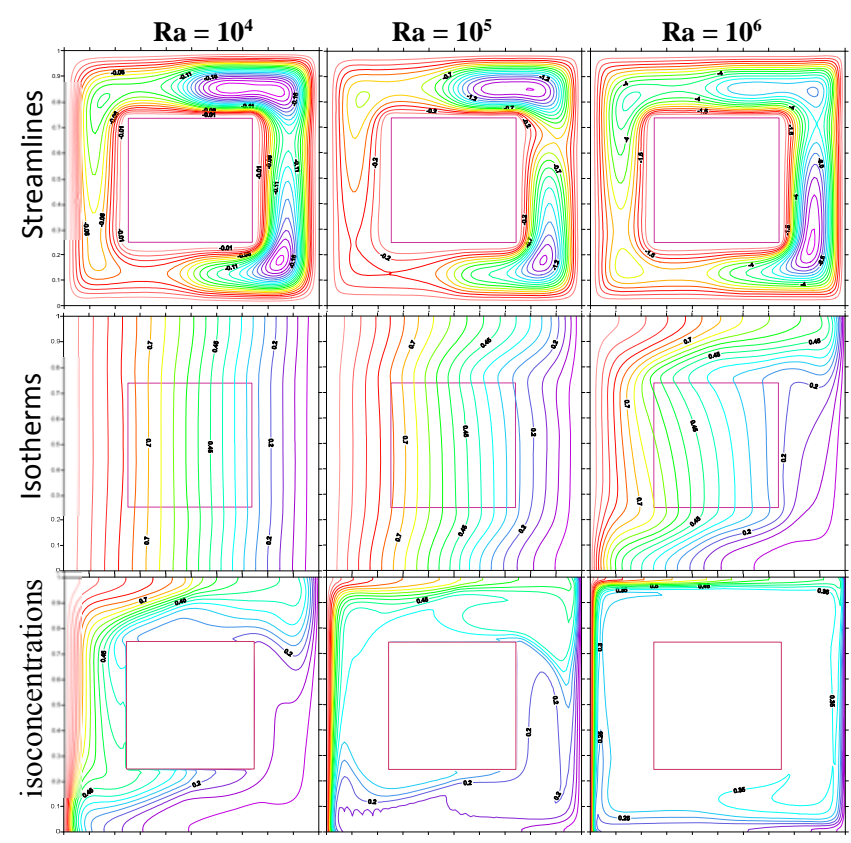

Fig. 7. Streamlines, isotherms, and isoconcentrations contours for selected values of Rayleigh number, at $L e=10, N=1, D a=10^{-3}, A=$ $0.5, K r=1$

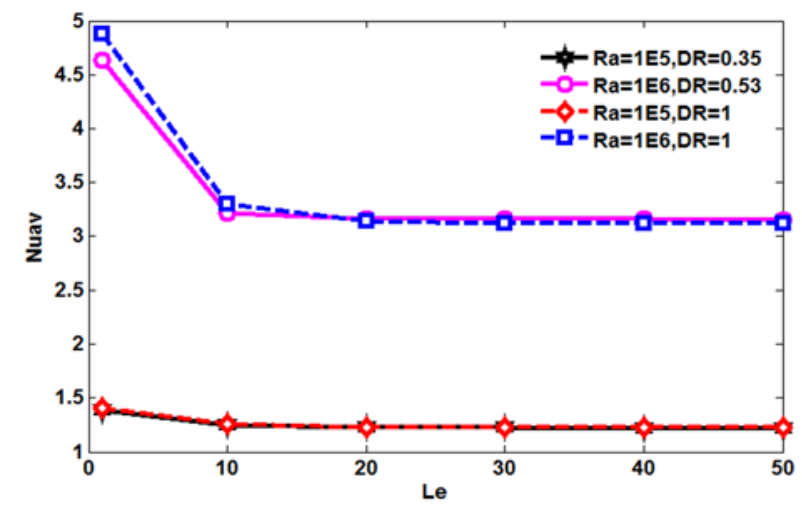

Fig. 8. Effect of Le number for two values of Diffusivity ratio on the average Nusselt number for $R a=10^{5}, 10^{6}$ and $N=1$.

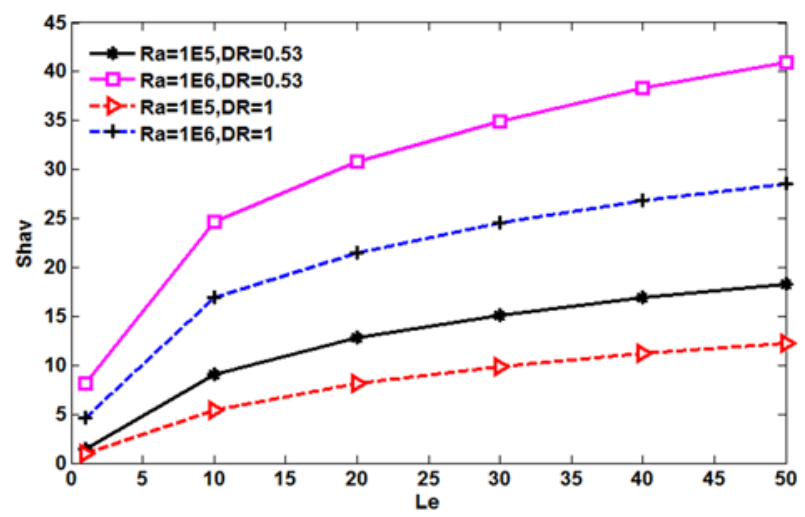

Fig. 9. Effect of Le number for two values of Diffusivity ratio on the average Sherwood numberfor $R a=10^{5}, 10^{6}$ and $N=1$. 


\section{Conclusions}

In-house FORTRAN program has been built in the present paper to study the double-diffusive natural convection inside a halvedlayered porous cavity with inner square conductive body. The study has been achieved numerically using the finite difference method with up-wind scheme procedure. The results have led to the following conclusions

1- The calculated mass diffusivity ratio, which takes into account non-unity tortuosity $\left(\mathrm{D}_{\text {eff }} / \mathrm{D}=0.53\right)$ ratio has a significant effect on the mass transfer in all studied Rayleigh and Lewis numbers. That is, the calculated Sherwood number according to $D_{\text {eff }} / \mathrm{D}=0.53$ is much larger than that calculated assuming a conventional ratio of $\mathrm{D}_{\text {eff }} / \mathrm{D}=1.0$. On the other hand, the convective heat transfer has bit affected by this ratio. That is Nusselt number is slightly less in the case of the present calculated mass diffusivity ratio, and only at higher Rayleigh number $\left(\mathrm{Ra}=10^{6}\right)$

2- The mass transfer is minimal when the buoyancy ratio equals to -0.5 , otherwise, the mass transfer increases with increasing the absolute buoyancy ratio.

3- The mass transfer is a continuously increasing function of Lewis number, while the convective heat transfer decreases with Lewis number to $\mathrm{Le}=10$.

4- The convective heat transfer is an increasing function of Rayleigh number, and as a result, the mass transfer follows this fashion.

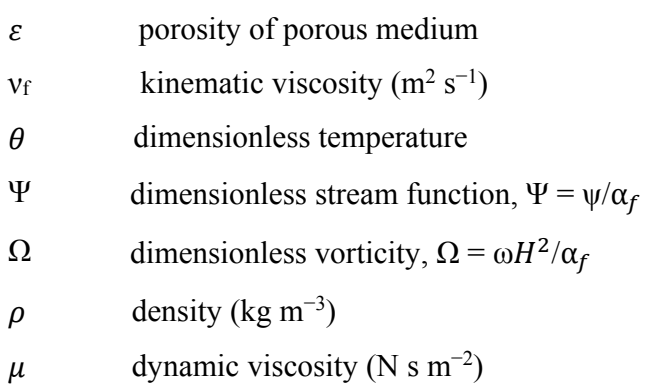

\section{Subscripts}

$\begin{array}{ll}\text { av } & \text { Average } \\ \text { b } & \text { Body } \\ \text { c } & \text { cold } \\ \text { eff } & \text { effective } \\ \text { f } & \text { fluid } \\ \text { h } & \text { high, hot } \\ \text { l } & \text { low } \\ \text { s } & \text { solid }\end{array}$

\section{References}

[1] D. B. Ingham, and I. Pop (Eds.), Transport Phenomena in Porous Media II, 1st ed., Pergamon, 2002

[2] A.C. Baytas, A.F. Baytas, D.B. Ingham and I. Pop, "Double Diffusive Natural Convection in an Enclosure Filled with a Step Type Porous Layer: NonDarcy Flow" International Journal of Thermal $\begin{array}{lll}\text { Sciences } & 48, & 665-673,\end{array}$ DOI:10.1016/j.ijthermalsci.2008.06.001

[3] S. C. Hirata - B. Goyeau · D. Gobin, "Stability of Thermosolutal Natural Convection in Superposed Fluid and Porous Layers" Transp. Porous Media 78, 525-536, 2009. DOI 10.1007/s11242-008-9322-9

[4] A.W. Islam, A.R. Sharif and E. S. Carlson, "Numerical Investigation of Double Diffusive Natural Convection of $\mathrm{CO} 2$ in a Brine Saturated Geothermal Reservoir" Geothermics 48, 101111,2013.DOI.org/10.1016/j.geothermics.2013.07.00 1

[5] N. Hadidi, R. Bennacer and Y. Ould-amer, "TwoDimensional Thermo-Solutal Natural Convective Heat and Mass Transfer in a Bi-Layered and Inclined Porous Enclosure" Energy 93, 2582-2592, 2015 DOI.org/10.1016/j.energy.2015.10.121

[6] M. Mharzi, M. Daguenet and S. Daoudi, "Thermosolutal Natural Convection in a vertically layered fluid-porous medium Heated from the side" Energy Conversion and Management 41, 1065-1090, 2000. DOI.org/10.1016/S0196-8904(99)00132-6

\footnotetext{
$\alpha \quad$ thermal diffusivity $\left(\mathrm{m}^{2} \mathrm{~s}^{-1}\right)$

$\beta \quad$ thermal expansion coefficient $\left(\mathrm{K}^{-1}\right)$
} 
[7] R. Bennacer, H. Beji and A.A. Mohamad, " Double Diffusive Convection in a vertical Enclosure inserted with two saturated porous layers confining a fluid layer" International Journal of Thermal Sciences 42, 141-151, 2003. DOI:10.1016/S1290-0729(02)00014$\underline{5}$

[8] D. Gobin, B. Goyeau and A. Neculae, "Convective Heat and solute Transfer in Partially porous cavities" International Journal of Heat and Mass Transfer 48, 1898-1908, 2005 DOI.org/10.1016/j.ijheatmasstransfer.2004.12.016

[9] Y. Outaleb, K. Bouhadef, and O. Rahli, "Double Diffusive Convection in a Partially Porous Cavity under Suction/Injection Effects" World Academy of Science, Engineering and Technology 55, 2011.

[10] N. Hadidi, Y. Ould-Amer and R. Bennacer, "Bilayered and inclined porous collector: Optimum Heat and Mass Transfer " Energy 51, 422-430, 2013. DOI.org/10.1016/j.energy.2013.01.012

[11] O. Rahli, K. Bouhadef, "Double -Diffusive Natural Convection in a partially porous Square Enclosure Effect of the Inclination".

[12] N. Hadidi, R. Bennacer, "Three-Dimensional Double Diffusive Natural Convection across a Cubical Enclosure Partially Filled by Vertical Porous Layer" International Journal of Thermal Sciences 101, 143157,2016.DOI.org/10.1016/j.ijthermalsci.2015.10.02 $\underline{9}$

[13] J.M. House, C. Beckermann, T.F. Smith, "Effect of a Centered Conducting Body on Natural Convection Heat Transfer in an Enclosure ", Numerical Heat Transfer , Part A, 18, 213-225, 1990. DOI.org/10.1080/10407789008944791

[14] M. Y. Ha, M. J. Jung, "A Numerical Study on ThreeDimensional Conjugate Heat Transfer of Natural Convection and Conduction in a Differentially Heated Cubic Enclosure with a Heat-generating Cubic Conducting Body " International Journal of Heat and Mass Transfer 43, 4229-4248, 2000. DOI.org/10.1016/S0017-9310(00)00063-6

[15] J. R. Lee, M. Y. Ha, "A Numerical Study of Natural Convection in a Horizontal Enclosure with a Conducting Body" International Journal of Heat and Mass Transfer 48, 3308-3318, 2005. DOI.org/10.1080/10407782.2013.784679
[16] E. J. Braga, M.J.S. de Lemos, "Heat Transfer in Enclosure s Having a Fixed Amount of Solid Material Simulated with heterogeneous and homogeneous models" International Journal of Heat and Mass Transfer 48, 4748-4765, 2005. DOI.org/10.1016/j.ijheatmasstransfer.2005.05.016

[17] E.J. Braga and M.J.S. de Lemos, "Laminar Natural Convection in Cavities Filled with Circular and Square Rods" International Communications in Heat and Mass Transfer 32, 1289-1297, 2005. DOI.org/10.1016/j.icheatmasstransfer.2005.07.014

[18] A.A. Merrikh and J.L. Lage, "Natural Convection in an Enclosure with Disconnected and Conducting Solid Blocks" International Journal of Heat and Mass Transfer 48, 1361-1372, 2005. DOI.org/10.1016/j.ijheatmasstransfer.2004.09.043

[19] M.K. Das and K. S. K. Reddy, "Conjugate Natural Convection Heat Transfer in an Inclined Square Cavity Containing a Conducting block" International Journal of Heat and Mass Transfer 49, 4987-5000, 2006.DOI.org/10.1016/i.ijheatmasstransfer.2006.05.0 $\underline{41}$

[20] F. Zhao, D. Liu and G. Tang, "Application Issues of the Streamline, Heat Line and Mass Line for Conjugate Heat and Mass Transfer" International Journal of Heat and Mass Transfer 50, 320-334, 2007.DOI.org/10.1016/j.ijheatmasstransfer.2006.06.0 $\underline{26}$

[21] F. Zhao, D. Liu and G. Tang, "Conjugate Heat Transfer in Square Enclosure" Heat Mass Transfer 43, 907-922, 2007. DOI:10.1007/s00231-006-0136-4

[22] Y. Varol, "Natural Convection in porous Triangular Enclosure with a Centered Conducting Body " International Communications in Heat and Mass Transfer 38, 368-376, 2011. DOI.org/10.1016/j.icheatmasstransfer.2010.12.013

[23] A.J. Chamkha, M.A. Ismael, "Natural convection in differentially heated partially porous layered cavities filled with nanofluid", Numerical Heat Transfer Part A, $\quad 65 \quad$ (2014) 1089-1113 DOI.org/10.1080/10407782.2013.851560

[24] Sheremet M.A. and Trifonova T.A., "Unsteady Conjugate Natural Convection in a Vertical Cylinder Containing a Horizontal Porous Layer: Darcy Model and Brinkman-Extended Darcy Model", Trans. Porous Med. 101 (2014) 437-463. DOI: 10.1007/s11242013-0253-8 